\title{
Evaluation of Risk Stratification Markers and Models in Acute Pulmonary Embolism: Rationale and Design of the MARS-PE (Mainz Retrospective Study of Pulmonary Embolism) Study Programme
}

\author{
Karsten Keller ${ }^{1,2, *}$, Johannes Beule ${ }^{3}$, Jörn Oliver Balzer ${ }^{4,5}$, Wolfgang Dippold ${ }^{3}$
}

\section{ABSTRACT}

An acute pulmonary embolism (PE) is a crucial event in patients' life and connected with serious morbidity and mortality. Regarding a high case-fatality rate, early and accurate risk-stratification is crucial. Risk for mortality and complications are closely related to hemodynamic stability and cardiac adaptations. The currently recommended risk-stratification approach is not overall simple to use and might delay the identification of those patients, who should be monitored more closely and may treated with more aggressive treatment strategies. Additionally, some risk-stratification criteria for the imaging procedures are still imprecise. Summarized, the search for the most effective risk-stratification tools is still ongoing and some diagnostic criteria might have to be refined.

In the MAinz Retrospective Study of Pulmonary Embolism (MARS-PE), overall 182 consecutive patients with confirmed PE were retrospectively included over a 5-year period. Clinical, echocardiographic, functional and laboratory parameters were assessed. The study was designed to provide answers to some of the mentioned relevant questions.

\section{KEYWORDS}

pulmonary embolism; study design; risk stratification; symptoms; outcome

AUTHOR AFFILIATIONS

${ }^{1}$ Center for Thrombosis and Hemostasis, University Medical Center Mainz, Johannes Gutenberg-University Mainz, Germany

${ }^{2}$ Center of Cardiology, Department of Cardiology I, University Medical Center Mainz, Johannes Gutenberg-University Mainz, Germany

${ }^{3}$ Department of internal medicine, St. Vincenz and Elisabeth Hospital Mainz (KKM), Mainz, Germany

${ }^{4}$ Department of Diagnostic and Interventional Radiology, Catholic Clinic Mainz (KKM), Mainz, Germany

${ }^{5}$ Department of Diagnostic and Interventional Radiology, University Clinic, Johann Wolfgang Goethe-University Frankfurt/Main, Frankfurt, Germany

* Corresponding author: Center for Thrombosis and Hemostasis (CTH), University Medical Center Mainz, Johannes Gutenberg-University Mainz, Langenbeckstr. 1, 55131 Mainz, Germany; e-mail: Karsten.Keller@unimedizin-mainz.de

Received: 28 November 2017

Accepted: 9 July 2018

Published online: 12 December 2018

Acta Medica (Hradec Králové) 2018; 61(3): 93-97

https://doi.org/10.14712/18059694.2018.124

(c) 2018 The Authors. This is an open-access article distributed under the terms of the Creative Commons Attribution License (http://creativecommons.org/licenses/by/4.0), which permits unrestricted use, distribution, and reproduction in any medium, provided the original author and source are credited. 


\section{INTRODUCTION}

An acute pulmonary embolism (PE) is a crucial event in a patients' life accompanied by serious morbidity and death worldwide $(1,2)$. Annual PE incidence has been reported ranging between 23 and 69 cases per 100,000 people in the general population (3).

Clinical presentation of acute PE comprises a wide spectrum from asymptomatic incidental finding to typical symptoms such as chest pain, dyspnoea, hemoptysis, collapse, syncope that may accompanied by hypotension, right heart failure, right heart infarction, cardiogenic shock, resuscitation and sudden cardiac death $(2,4-6)$. Regarding a high case fatality rate ranging between $1 \%$ and $60 \%(4,7)$, promptly, early and accurate risk stratification in respect to adverse outcome of patients with an acute $\mathrm{PE}$ event is crucial $(2,6,8,9)$.

Pathophysiologically, PE is caused by thrombotic material occluding the pulmonary arteries (10-12). If occlusion affects more than $30-50 \%$ of the pulmonary arterial bed, hemodynamically consequences of acute PE have to be expected. Right ventricle (RV) under normal conditions has a narrow range to handle an acute afterload increase. An abrupt afterload increase, seen in acute PE events, frequently results in RV overload with an elevated RV wall tension and right ventricular dysfunction (RVD) (10, 1218). Depending on PE severity and intensity of right heart overload, RVD, coronary under-perfusion, decline of cardiac output, myocardial injury, cardiac shock, right heart failure and PE-related death could be the results (12-14, $16,19,20)$.

Risk for mortality and complications in acute PE events are closely related to (initial) hemodynamic stability as well as cardiac adaptations (12-17, 21-24). Therefore, a risk-related classification of acute PE severity has been recommended to guide risk-adjusted management of patients; the short-term adverse outcome of acute PE is dependent on PE severity status stratified by clinical findings during the acute phase, results of imaging procedures and biomarker measurements indicating for RVD, but also on the factors age and comorbidities (determining the reserve capacity of the cardio-pulmonary system) $(2,12)$. Haemodynamically unstable PE patients are classified as high-risk PE $(2,12)$ or massive PE $(9)$, with high mortality rate ( $>15 \%$ in the first 30 days after $P E$ event) $(11,12,16$, 21). Haemodynamically stable PE patients (non-high-risk or non-massive PE patients) can be subdivided into those with RVD and/or positive biomarkers, such as cardiac troponin (cTn) and/or brain natriuretic peptides (BNP), and those without both $(11,12)$. Haemodynamically stable PE patients with RVD or positive biomarker levels (submassive PE stadium) show an intermediate risk, with a short term mortality of $3-15 \%(10,12,16,21)$. Haemodynamically stable PE patients without RVD and without an elevated biomarker levels (low-risk PE) reveal the best prognosis, with a short term mortality rate $<2 \%(10-12,14,21)$. During the past years, recommendations for PE risk stratification were adapted, because it became obvious that laboratory biomarkers alone were not sufficient for risk stratification (12). Several risk stratification markers, algorithms and scores have been developed to identify those patients, who are at higher risk for the development of adverse events and especially mortality $(2,25)$.

Therefore, PE classification approach was refined and improved by the (simplified) Pulmonary Embolism Severity Index (PESI) implemented in the recommendations of the 2014 ESC guideline (2). Summarized, risk stratification comprises the factors age, gender, symptoms, clinical examination markers such as temperature, altered mental status, hypoxia, respiratory rate, blood pressure and heart rate, comorbidities, VTE risk factors, as well as biomarkers including especially markers of heart strain and myocardial injury and imaging procedures focusing on the adaptations of the heart and the burden of PE (2).

Several diagnostic tools and biomarkers are already in use for early risk stratification in non-high-risk PE patients. Especially, biomarker testing can improve inpatient management, enabling better outcomes in acute PE (21). cTn and BNP are well established markers for risk stratification in acute $\mathrm{PE}$, and their elevations are closely connected with an increased mortality rate (11-18, 22, 24, 26-28). Besides these two biomarkers, several other biomarkers have been identified for prediction of poor outcome $(9,12,21,29)$.

More than $80 \%$ of the PE patients are normotensive (30,31), and between $25 \%$ and $55 \%$ of the normotensive $\mathrm{PE}$ patients have an identified RVD in echocardiographic or computer-tomographic examinations $(30,32)$. RVD in hemodynamically stable PE patients appears to alter patients' prognosis significantly (30) and right ventricular failure (RVF) with RVD are the most common causes of death in the first 30 days after the PE index event (33). Therefore, morphologic adaptations of the heart identified in imaging procedures are important findings in risk stratification process. Especially, the prognostic value of echocardiography for risk stratification in hemodynamically stable PE patients was confirmed in several studies (30$36)$. Echocardiography is currently the mainstay examination for assessment of RVD in patients with PE $(32,37)$. Although several studies confirmed that RVD is connected with higher rates of death, recurrent $P E$ events and complications $(9,12,30,32-34,36)$, definitions of PE-induced RVD criteria vary markedly in different studies $(30-32,37)$ and recommendations for detection of RVD in the guidelines for the echocardiographic criteria for the assessment of RVD in acute PE are not precisely defined $(2,9,12)$.

Another often used examination tool is the electrocardiography (ECG). In general, all patients with chest pain, dyspnoea, syncope or collapse should obtain an ECG in the emergency department immediately after first medical contact (38-40). This ECG is primarily used to exclude an acute ST-segment elevation myocardial infarction and arrhythmias $(38,39)$, but is also an important diagnostic tool in PE $(40,41)$.

Although this study focus on the in-hospital course of the PE patients and PE has traditionally been assumed as acute disease, also the long-term course of PE survivors can be complicated by recurrent venous thromboembolism events (VTE), development of post-thrombotic syndrome after accompanying deep venous thrombosis (DVT), chronic thromboembolic pulmonary hypertension (CTEPH), and treatment complications such as bleeding 
events caused by anticoagulant therapy and might be accompanied by a higher risk for artherosclerotic events (42-45). Despite these established long-term sequelae, studies have consistently demonstrated, that approximately $1 / 2$ of the patients suffer from functional limitations or decreased QoL and rare CTEPH seems to be only the most extreme expression (46).

\section{UNANSWERED QUESTIONS IN THE ACUTE PHASE OF PE AND OBJECTIVES OF THIS STUDY}

An acute PE is a frequent cause of death and serious disability (2). Risk of adverse outcome during the in-hospital phase varies widely depending on the clinical severity and presence of RVD and elevated biomarkers $(2,25)$.

The currently recommended risk stratification approach is in part not simple to use and might delay the resulting risk classification and identification of those patients, who should be monitored more closely and might undergo more aggressive treatment strategies such as systemic thrombolysis. Although it is widely easy to identify the high-risk PE patients, further risk classification in normotensive PE patients depends on imaging procedure, biomarkers and the sPESI or PESI according to the ESC guideline of the year 2014 (2). This approach for risk stratification in normotensive patients might delay the risk classification and is complicated for physicians in the emergency room with small time slots for each patient.

Additionally, some of the criteria, especially for the imaging procedures, remained imprecise.

Therefore, the search for the most effective risk stratification tools is still ongoing and diagnostic imaging criteria might have to be refined.

The MAinz Retrospective Study of Pulmonary Embolism (MARS-PE) study programme (Figure 1) for the evaluation of risk stratification process in acute PE enrolled consecutive patients with confirmed PE retrospectively over a 5-year period. Clinical, echocardiographic, functional and laboratory parameters were assessed. MARS-PE has been designed to provide answers to the above relevant remaining questions regarding morbidity and mortality after PE.

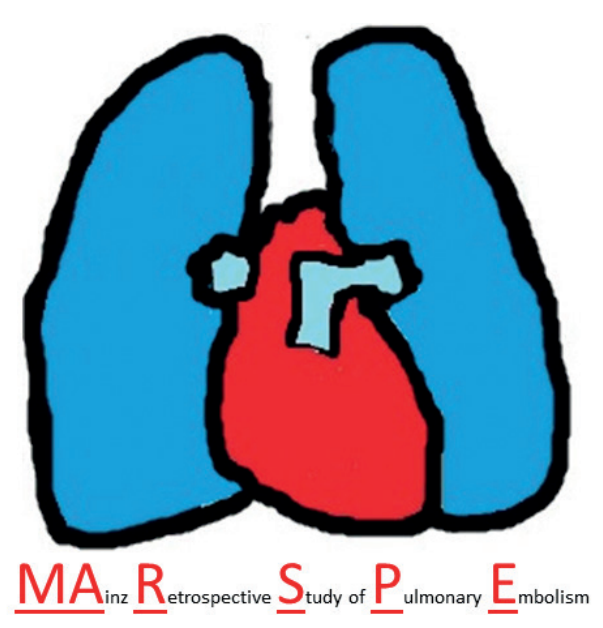

Fig. 1 MAinz Retrospective Study of Pulmonary Embolism (MARS-PE) Logo.

\section{STUDY POPULATION}

A total of 182 consecutive patients with acute, objectively diagnosed PE were retrospectively included in MARS-PE on the basis of the eligibility criteria listed in Table 1. The MARS-PE aimed to include PE all-comers with PE, irrespective of clinical severity, RVD, and size or extent of pulmonary emboli. All included patients were treated at the Department of Internal Medicine of the St. Vincenz and Elisabeth hospital of the Catholic Clinic Mainz (Mainz, Germany) between May 2006 and June 2011. The patients were found by a search of the hospital information system database for the diagnostic code of PE (ICD-Code: I26).

All CT, scintigraphic and phlebography images were analysed by experienced radiologists. If the diagnosis of $\mathrm{PE}$ was not confirmed by the criteria above, the patient was not included in this study.

Tab. 1 Eligibility criteria.

\begin{tabular}{|l|l|}
\hline Inclusion Criteria & Exclusion Criteria \\
\hline - Objectively confirmed & - Patients in whom the diagno- \\
diagnosis of acute PE by & sis of PE was not confirmed \\
identified filling defect in the & by the examinations \\
pulmonary artery system & - Patients younger than \\
on a computed tomography & 18 years old \\
pulmonary angiogram (CT) & - Previous enrollment in this \\
of the chest, a scintigraphic & study \\
ventilation-perfusion (V/Q) & \\
scan read as high probability & \\
for PE or positive venous & \\
ultrasound/phlebography & \\
of an extremity consistent & \\
with deep venous thrombosis & \\
(DVT) in patients with typical & \\
symptoms of PE (chest pain & \\
or dyspnoea) and positive & \\
D-dimer & \\
- Age $\geq 18$ years \\
- Patients were treated in \\
the Department of Internal \\
Medicine in the St. Vincenz \\
and Elisabeth hospital of \\
the Catholic Clinic of Mainz, \\
Mainz, Germany \\
\hline
\end{tabular}

\section{DEFINITIONS}

\section{MYOCARDIAL INJURY}

According to the AHA scientific statement from 2011, myocardial necrosis was defined as a cardiac troponin I (cTnI) elevation $>0.4 \mathrm{ng} / \mathrm{ml}(9)$.

\section{RIGHT VENTRICULAR DYSFUNCTION (RVD)}

RVD was defined according to the AHA scientific statement (9) as a quotient of right ventricular (RV) septal-lateral diameter / left ventricular (LV) septal-lateral diameter $>0.9$ in the four-chamber view on transthoracic echocardiography or CT (9). Moreover, the RVD was defined as RV hypokinesis and tricuspid regurgitation by echocardiography (9). For some analyses a sPAP $>30 \mathrm{mmHg}$ was additionally included in the RVD criteria. 
HIGH-RISK PE SEVERITY STATUS

PE patients with a systolic blood pressure $<90 \mathrm{mmHg}$ at admission were classified as high-risk $\mathrm{PE}$ according to the definition from the recent and current ESC guidelines (2, 12) and the AHA scientific statement (9).

\section{INTERMEDIATE-RISK (= SUBMASSIVE) PE SEVERITY STATUS}

According to the recent, but at this time valid ESC guidelines from 2008 on the diagnosis and management of acute pulmonary embolism (12) and the AHA scientific statement for management of massive and submassive PE, iliofemoral deep vein thrombosis, and chronic thromboembolic pulmonary hypertension from 2011 (9) the nonhigh-risk PE patients were subdivided in 2 groups with regard to RVD and cTnI level. PE patients with RVD or pathological cTnI levels were included in the submassive PE group with intermediate risk. Patients without RVD and without elevated troponin levels were classified as non-massive PE group with low risk.

\section{STUDY PARAMETERS}

The retrospective analysis of $\mathrm{PE}$ patients focused on anamnesis with medical history as well as clinical, laboratory, ultrasound, echocardiographic and CT examination results.

\section{PATIENT OUTCOMES}

We analyzed the study outcome parameters in all included PE patients or in those non-high-risk PE patients, who were hemodynamically stable (normotensive) and among these, especially in those normotensive patients with an accurate transthoracic echocardiography, as appropriate.

The proposed study endpoints comprised the following: i) all-cause in-hospital mortality as well the surrogate markers ii) RVD, iii) myocardial injury and iv) PE severity status according to the ESC guidelines from 2008 (12). The study endpoints of RVD, myocardial injury as well as the PE severity status according to the ESC guidelines from 2008 (12) are established surrogate markers of poorer outcome in the acute course after the PE event and therefore, were chosen as proposed study endpoint parameters.

\section{STATISTICAL ANALYSIS}

Descriptive statistics for the relevant baseline comparisons of the respective baseline-groups were provided with mean \pm standard deviation, median and interquartile range (IQR), depending on Gaussian or skewed distribution, or absolute numbers and corresponding percentages. Baseline variables of the groups were compared using the Wilcoxon-Whitney U test, the Students' T-Test or in categorical variables with Fisher's exact or $\mathrm{Chi}^{2}$ test, as appropriate.
We calculated uni-variate and (if necessary to test the independence) multi-variate logistic regression models to examine the associations between risk stratification markers and the study outcome markers.

Receiver operating characteristic (ROC) curves with areas under the curves (AUC) and Youden indices were calculated to test the effectiveness of markers to predict outcome parameters in acute PE. The Wilcoxon-Mann-Whitney test was used to test the deviation of the ROC curve from the angle bisector.

Although some of the statisticians recommend to calculate a post-hoc power analysis (47), we are in accordance with the majority of the statisticians of the opinion that a post hoc power analysis is inappropriate for the evaluation of study results $(48,49)$. Therefore, we did not calculate such a post-hoc power calculation.

The software SPSS ${ }^{\circledR}$ (version 22.0; SPSS Inc., Chicago, Illinois), $\mathrm{R}$ version 2.14.1 from $\mathrm{R}$ Development Core Team (2011) (R Foundation for Statistical Computing, Vienna, Austria) and the commercially available software BIAS ${ }^{\circledR}$ (version 10.04; epsilon press, Frankfurt, Germany) were used for computerized analysis. Only $\mathrm{P}$ values of $<0.05$ (two-sided) were considered to be statistically significant.

\section{ETHICAL ASPECTS}

The requirement for informed consent was waived as we used only anonymized retrospective data routinely collected during the health screening process. Studies in Germany involving a retrospective analysis of diagnostic standard data do not require an ethics statement.

The study was conducted in St. Vincenz and Elisabeth Hospital Mainz (KKM).

\section{REFERENCES}

1. Konstantinides SV, Barco S, Rosenkranz S, et al. Late outcomes after acute pulmonary embolism: rationale and design of FOCUS, a prospective observational multicenter cohort study. J Thromb Thrombolysis 2016; 42: 600-9.

2. Konstantinides SV, Torbicki A, Agnelli G, et al., Task Force for the $\mathrm{D}$ and Management of Acute Pulmonary Embolism of the European Society of C. 2014 ESC guidelines on the diagnosis and management of acute pulmonary embolism. Eur Heart J 2014; 35: 3033-69, 3069a-3069k.

3. Konstantinides S. Acute Pulmonary Embolism. N Engl J Med 2008; 359: 2804-13.

4. Agnelli G and Becattini C. Acute pulmonary embolism. N Engl J Med 2010; 363: 266-74.

5. Keller K, Beule J, Schulz A, Dippold W. Troponin I as risk stratification marker in acute pulmonary artery embolism. Phlebologie 2013; 42: 261-9.

6. Keller K, Beule J, Balzer JO and Dippold W. Typical symptoms for prediction of outcome and risk stratification in acute pulmonary embolism. Int Angiol 2015.

7. Carson JL, Kelley MA, Duff A, et al. The clinical course of pulmonary embolism. N Engl J Med 1992; 326: 1240-5.

8. Sam A, Sanchez D, Gomez V, et al. The shock index and the simplified PESI for identification of low-risk patients with acute pulmonary embolism. Eur Respir J 2011; 37: 762-6.

9. Jaff MR, McMurtry MS, Archer SL, et al. Management of massive and submassive pulmonary embolism, iliofemoral deep vein thrombosis, and chronic thromboembolic pulmonary hypertension: a scientific statement from the American Heart Association. Circulation 2011; 123: $1788-830$. 
10. Chalikias GK, Tziakas DN, Stakos DA, Konstantinides SV. Managment of acute pulmonary embolism: a contemporary risk-tailored approach. Hellenic J Cardiol 2010; 51: 437-50.

11. Sanchez O, Trinquart L, Colombet I, et al. Prognostic value of right ventricular dysfunction in patients with haemodynamically stable pulmonary embolism: a systematic review. Eur Heart J 2008; 29: 1569-77.

12. Torbicki A, Perrier A, Konstantinides S, et al. Guidelines on the diagnosis and management of acute pulmonary embolism: the Task Force for the Diagnosis and Management of Acute Pulmonary Embolism of the European Society of Cardiology (ESC). Eur Heart J 2008; 29: 2276-315.

13. Hsu JT, Chu CM, Chang ST, et al. Prognostic role of alveolar-arterial oxygen pressure difference in acute pulmonary embolism. Circ J 2006; 70: 1611-6.

14. Goldhaber SZ. Assessing the prognosis of acute pulmonary embolism: tricks of the trade. Chest 2008; 133: 334-6.

15. Giannitsis E, Muller-Bardorff M, Kurowski V, et al. Independent prognostic value of cardiac troponin $\mathrm{T}$ in patients with confirmed pulmonary embolism. Circulation 2000; 102: 211-7.

16. Schellhaass A, Walther A, Konstantinides S, Bottiger BW. The diagnosis and treatment of acute pulmonary embolism. Dtsch Arztebl Int 2010; 107: 589-95.

17. Jimenez D, Diaz G, Molina J, et al. Troponin I and risk stratification of patients with acute nonmassive pulmonary embolism. Eur Respir J 2008; 31: 847-53.

18. Mikulewicz M, Lewczuk J. Importance of cardiac biomarkers in risk stratification in acute pulmonary embolism. Cardiol J 2008; 15: 17-20.

19. Keller K, Beule J, Schulz A, Coldewey M, Dippold W, Balzer JO. D-dimer for risk stratification in haemodynamically stable patients with acute pulmonary embolism. Adv Med Sci 2015; 60: 204-10.

20. Ribeiro A, Lindmarker P, Juhlin-Dannfelt A, Johnsson H, Jorfeldt L. Echocardiography Doppler in pulmonary embolism: right ventricular dysfunction as a predictor of mortality rate. Am Heart J 1997; 134: 479-87.

21. Ohigashi H, Haraguchi G, Yoshikawa S, et al. Comparison of biomarkers for predicting disease severity and long-term respiratory prognosis in patients with acute pulmonary embolism. Int Heart J 2010; 51: 416-20.

22. Jimenez D, Uresandi F, Otero R, et al. Troponin-based risk stratification of patients with acute nonmassive pulmonary embolism: systematic review and metaanalysis. Chest 2009; 136: 974-82.

23. Sanchez O, Trinquart L, Caille V, et al. Prognostic factors for pulmonary embolism: the prep study, a prospective multicenter cohort study. Am J Respir Crit Care Med 2010; 181: 168-73.

24. Wu AH, Jaffe AS, Apple FS, et al. National Academy of Clinical Biochemistry laboratory medicine practice guidelines: use of cardiac troponin and B-type natriuretic peptide or N-terminal proB-type natriuretic peptide for etiologies other than acute coronary syndromes and heart failure. Clin Chem 2007; 53: 2086-96.

25. Hobohm L, Hellenkamp K, Hasenfuss G, Munzel T, Konstantinides S, Lankeit M. Comparison of risk assessment strategies for not-highrisk pulmonary embolism. Eur Respir J 2016; 47: 1170-8.

26. Becattini C, Vedovati MC, Agnelli G. Prognostic value of troponins in acute pulmonary embolism: a meta-analysis. Circulation 2007; 116: 427-33.

27. Margato R, Carvalho S, Ribeiro H, Mateus P, Fontes P, Moreira JI. Cardiac troponin I levels in acute pulmonary embolism. Rev Port Cardiol 2009; 28: 1213-22.

28. Lankeit M, Jimenez D, Kostrubiec M, et al. Validation of N-terminal pro-brain natriuretic peptide cut-off values for risk stratification of pulmonary embolism. Eur Respir J 2014; 43: 1669-77.

29. Becattini C, Lignani A, Masotti L, Forte MB and Agnelli G. D-dimer for risk stratification in patients with acute pulmonary embolism. J Thromb Thrombolysis 2012; 33: 48-57.
30. Kreit JW. The impact of right ventricular dysfunction on the prognosis and therapy of normotensive patients with pulmonary embolism. Chest 2004; 125: 1539-45.

31. Konstantinides S, Goldhaber SZ. Pulmonary embolism: risk assessment and management. Eur Heart J 2012.

32. Becattini C, Vedovati MC, Agnelli G. Right ventricle dysfunction in patients with pulmonary embolism. Intern Emerg Med 2010; 5: 453-5.

33. Kucher N, Rossi E, De Rosa M, Goldhaber SZ. Prognostic role of echocardiography among patients with acute pulmonary embolism and a systolic arterial pressure of $90 \mathrm{~mm} \mathrm{Hg}$ or higher. Arch Intern Med 2005; 165: 1777-81.

34. Grifoni S, Olivotto I, Cecchini P, et al. Short-term clinical outcome of patients with acute pulmonary embolism, normal blood pressure, and echocardiographic right ventricular dysfunction. Circulation 2000; 101: 2817-22.

35. Vieillard-Baron A, Page B, Augarde R, et al. Acute cor pulmonale in massive pulmonary embolism: incidence, echocardiographic pattern, clinical implications and recovery rate. Intensive Care Med 2001; 27: 1481-6.

36. Fremont B, Pacouret G, Jacobi D, Puglisi R, Charbonnier B, de Labriolle A. Prognostic value of echocardiographic right/left ventricular end-diastolic diameter ratio in patients with acute pulmonary embolism: results from a monocenter registry of 1,416 patients. Chest 2008; 133: 358-62.

37. Keller K, Beule J, Schulz A, Coldewey M, Dippold W, Balzer JO. Right ventricular dysfunction in hemodynamically stable patients with acute pulmonary embolism. Thromb Res 2014.

38. Roffi M, Patrono C, Collet JP, et al. 2015 ESC Guidelines for the management of acute coronary syndromes in patients presenting without persistent ST-segment elevation: Task Force for the Management of Acute Coronary Syndromes in Patients Presenting without Persistent ST-Segment Elevation of the European Society of Cardiology (ESC). Eur Heart J 2015.

39. Steg PG, James SK and Gersh BJ. 2012 ESC STEMI guidelines and reperfusion therapy: Evidence-based recommendations, ensuring optimal patient management. Heart 2013; 99: 1156-7.

40. Keller K, Beule J, Balzer JO and Dippold W. Right bundle branch block and SIQIII-type patterns for risk stratification in acute pulmonary embolism. J Electrocardiol 2016; 49: 512-8.

41. Kucher N, Walpoth N, Wustmann K, Noveanu M, Gertsch M. QR in V1 - an ECG sign associated with right ventricular strain and adverse clinical outcome in pulmonary embolism. Eur Heart J 2003; 24: 1113-9.

42. Klok FA, Cohn DM, Middeldorp S, et al. Quality of life after pulmonary embolism: validation of the PEmb-QoL Questionnaire. J Thromb Haemost 2010; 8: 523-32.

43. Prandoni P. Links between arterial and venous disease. J Intern Med 2007; 262: 341-50.

44. Prandoni P. Venous thromboembolism and atherosclerosis: is there a link? J Thromb Haemost 2007; 5 Suppl 1: 270-5.

45. Hong C, Zhu F, Du D, Pilgram TK, Sicard GA, Bae KT. Coronary artery calcification and risk factors for atherosclerosis in patients with venous thromboembolism. Atherosclerosis 2005; 183: 169-74.

46. Klok FA, van der Hulle T, den Exter PL, Lankeit M, Huisman MV, Konstantinides S. The post-PE syndrome: a new concept for chronic complications of pulmonary embolism. Blood Rev 2014; 28: 221-6.

47. Rasch B, Friese M, Hofmann W, Naumann E. Quantitative Methoden. Heidelberg: Springer; 2014.

48. Levine M, Ensom MH. Post hoc power analysis: an idea whose time has passed? Pharmacotherapy 2001; 21: 405-9.

49. O’Keefe DJ. Brief Report: Post Hoc Power, Observed Power, A Priori Power, Retrospective Power, Prospective Power, Achieved Power: Sorting Out Appropriate Uses of Statistical Power Analyses. Communication Methods and Measures 2007; 1: 291-9. 